\title{
The Role of Psychosocial Factors in Wellbeing and Self-Care in Young Adults with Type 1 Diabetes
}

\author{
Renata Pires-Yfantouda ${ }^{1, *}$, Michael Evangeli $^{2}$ \\ ${ }^{1}$ Department Psychology, Doctorate in Health Psychology, City University, London, EC1V 0HB, UK; Disability Medicine, Rheumatology, \\ Amersham Hospital, HP7 0JD \\ ${ }^{2}$ Department of Psychology, Doctorate in Clinical Psychology, Royal Holloway, University of London, Egham Hill, Egham, Surrey, TW20 \\ OEX
}

\begin{abstract}
The purpose of this study is to explore the relationship between glycaemic control, locus of control beliefs, diabetes knowledge and wellbeing in young adults with type 1 diabetes. A cross-sectional study of forty-two young adults (16-25 years) with type 1 diabetes recruited from two diabetes clinics in London. Participants completed postal questionnaires designed for this specific population. Wellbeing was assessed by the W-B12, locus of control by the ADDLoC and diabetes knowledge by the ADKnowl. HbA1c was employed as a measure of glycaemic control. Results indicated that external (health professionals) and internal locus of control beliefs and diabetes knowledge were significantly associated with psychological wellbeing. Patients place high expectations on their practitioners and accordingly, practitioners need to address patients' diabetes knowledge to help them to manage their diabetes effectively and independently. The relationship between internal and external locus of control beliefs, diabetes knowledge and wellbeing indicates the importance of addressing empowerment and self-efficacy in psychoeducation interventions for this client group.
\end{abstract}

Keywords Type 1 Diabetes, Wellbeing, Self-Care, Psychosocial

\section{Introduction}

Type 1 diabetes is rapidly increasing worldwide amongst young people and it is predicted that 76,000 will develop the condition every year (International Diabetes Federation, IDF, 2009). Life expectancy is reduced by at least fifteen years for someone with Type 1 diabetes. Diabetes care represents a high percentage of all health care costs, comprising approximately $5 \%$ of the total UK National Health Service expenditure (and up to 10\% of hospital in-patient expenditure) (The Department of Health (DoH), 2007; 2003; The National Institute of Clinical Excellence (NICE), 2004).

To manage type 1 diabetes, patients need to maintain strict monitoring of their blood glucose levels and to take insulin. The goals of treatment are to maintain blood glucose levels as close to the normal range as possible to reduce the risk of diabetes-related complications. It is recognised that achieving ideal metabolic control is not a straight-forward task because strict monitoring of one's diabetes is likely to impact on individuals' well-being and quality of life (Wolpert \& Anderson, 2001; Bradley, 1996).

Glycosylated haemoglobin or $\mathrm{HbA} 1 \mathrm{C}$ is primarily used as a treatment-tracking test reflecting average blood glucose

* Corresponding author:

r.pires@city.ac.uk (Renata Pires-Yfantouda)

Published online at http://journal.sapub.org/diabetes

Copyright (C) 2012 Scientific \& Academic Publishing. All Rights Reserved levels over the preceding 90 days (Bradley, 1996). In spite of variations according to patients' age and body max index (BMI), on average a HbA1C level of between 6.5 and $7.0 \%$ is considered good (The National Diabetes Education Program (NDEP), 2007).

According to Woodcock and Bradley (2007), in addition to biomedical outcomes and processes, a profile of psychological outcomes is also required to understand diabetes control and complications. Measures of psychological processes such as diabetes-related knowledge, self-care skills, locus of control, and psychological well-being can indicate appropriate interventions Improving psychological well-being may entail, in the short-term, reducing feelings of anxiety and fatigue, and, in the longer term, reducing the risks of complications that can impact on psychological well-being (Bradley, 1994).

\subsection{Psychological Wellbeing}

Psychological wellbeing is a generic term which has been broadly defined and used in the literature. Wellbeing has been used to refer to an absence of depression, satisfaction with life as a whole and with reference to specific domains, for example, self-esteem, perceived social support, perception of control and values (González, Casas, \& Coenders, 2007).

A significant limitation in the well-being literature is that many studies look at negative domains of well-being and limit themselves to revealing factors associated with psy- 
chological morbidity. Even though these are important factors to be considered, they fail to address factors that increase resilience and help patients to cope with the challenges of having a chronic condition. Furthermore, a number of studies employ scales which confound with somatic symptoms (Jacobson, 1993).

Physiological and psychological changes during puberty can affect diabetes management and the onset of psychiatric problems (Kakleas, Kandyla, Karayianni \& Karavanaki, 2009). Recent studies shown that diabetes education programmes and health promotion strategies improve symptoms of depression, diabetes knowledge, glycaemic control, self-care and self-efficacy (Kluding, 2010; Castillo, 2010). Education interventions are easy to implement and are likely to have a very positive effect on diabetes knowledge and management.

\subsection{Diabetes Knowledge}

The UK Prospective Diabetes Study Group (UK Prospective Diabetes Study Group, 1998) provided considerable evidence to indicate how diabetes complications can be prevented or delayed; indicating improving patients' knowledge is a key task. They highlighted the features of good patient education, which included a continuous evaluation of patients' knowledge. Diabetes education is invaluable at as a preventative strategy: A recent study with low income Hispanic families whereby a diabetes prevention programme delivered in a school setting was effective in increasing diabetes-related knowledge, chronic disease awareness, and self-reported healthy behaviour (Coleman, 2010). Even though diabetes knowledge is essential as a preventative measure and also for patients to make appropriate decisions about their regimen the diabetes literature has not shown always consistent association between knowledge and good diabetes self-management (Anderson, \& Rubin, 1996). A key factor to be considered is how knowledge is transferred from practitioners to patients. In a study by Bogner (2010) in which a serious of education interventions were conducted by a health professional in interaction with diabetes patients, the interaction served as a basis for behavioural changes and transformative learning in 4 out of 5 clients. According to Anderson \& colleagues (1996) diabetes knowledge, understanding and beliefs may be incompatible with self-care in some instances; the recommendations made may create conflicts with other priorities in the individual's life impacting their quality of life and wellbeing. By devising a good therapeutic alliance with patients, health professionals can intervene by providing education, increasing diabetes knowledge and patients' ability to make choices. By feeling more self-efficacious about diabetes management it is likely that patients take more responsibility over their treatment resulting in an increase in internal locus of control beliefs.

\subsection{Locus of Control and Diabetes}

Locus of control beliefs refer to a relatively stable set of beliefs, held by an individual about the likely causal relationship between their actions, and those of others, and the outcomes of events and situations (Walker, 2004). Health locus of control has been shown to be related to whether an individual changes their behaviour and to the kind of communication style they require from health professionals (Ogden, 2004)

Locus of control has been recognized as an important domain in diabetes and it is important to explore these beliefs to help diabetes patients to self-care. There is a question of whether locus of control beliefs might play a role in one's actively seeking diabetes knowledge and adhering to education provided by practitioners

Feelings of personal control are also likely to affect one's well-being, amongst other psychological variables (Bradley, 1994).

A number of studies found that internal locus of control played a role in diabetes management (Bradley, 1994). External locus of control has been more frequently associated with poor diabetes control. A more recent study found a relationship between compliance, social support and both external (health professionals) and internal locus of control beliefs (Maltby, Day, \& Macaskill, 2007)

Further research is needed exploring the relationship between internal and external locus of control, diabetes management and wellbeing. However, a recent meta-analysis Gherman et al., 2011) only found a weak correlation between powerful others and chance LoC and diabetes control.

In summary, to-date the diabetes literature has explored the relationship between negative domains of psychological wellbeing and single factors. The literature is not conclusive in relation to internal and external locus of control domains and its relationship to other psychological variables and diabetes management. By increasing diabetes knowledge and education amongst patients it is possible that the ability to make choices and internal locus of control beliefs will increase and this might affect wellbeing and diabetes management.

The aims of the current study are to explore the relationship between wellbeing, diabetes knowledge and locus of control beliefs in young adults with type 1 diabetes.

It was hypothesised that participants' levels of well being would be related to greater knowledge of their condition, higher locus of control beliefs. The relationship between some but not all of these variables has been established in previous research (Arraras et al., 2002; Elfström \& Kreuter, 2006).

\section{Methods}

The current study invited adolescents and young adults (16 to 25 years old) who were diagnosed at least a year ago with type 1 diabetes to take part in this study. This age group was targeted as there were available diabetes clinics dedicated to patients within the age ranges a) $14-18$ and b) $18-25$ years-old. These clinics were developed in recognition that 
younger adults with type 1 diabetes have specific needs which can be targeted at the early onset of their illness.

The exclusion criterion was not having the ability to read English and having been diagnosed with type 1 diabetes for less than a year. The current study recruited participants from two north-west London hospitals with dedicated services for younger people with type 1 diabetes.

\subsection{Measures and Summary}

1. Socio-demographics and $\mathrm{HbAlC}$ levels were taken from the medical notes and computerised databases. HbA1C was measured using DCCT aligned. Regimen information and factors associated with general health were taken from a short-questionnaire.

2. Diabetes Related Knowledge (ADKnowl) (Speight \& Bradley, 2001) was designed to measure essential knowledge of diabetes and its management. It targets knowledge deficits which can be related to measurable outcomes. It includes 27 item-sets (114 items) measuring diabetes treatment, management of diabetes when ill (separate sets for insulin treatment and tablets), causes of symptoms and actions to be taken during hypoglycaemia episodes, diet and food, effects of physical activity, effects of smoking and alcohol, reducing the risk of developing diabetes complications, and foot-care. It includes 104 items measuring diabetes treatment and management. A reliability analysis was conducted for this scale showing a moderate level of reliability for the ADKnowl $(\alpha=.60)$.

3. Diabetes Control (ADDLoC) (Bradley, 1994). The audit of Diabetes-Dependent Locus of Control balances items across four subscales (internality - i.e. "It's within my power to achieve acceptable diabetes control"; significant others i.e. "If I am to have a good quality of life as well as control of my diabetes, I need the support of those around me"; medical control and chance). The subscales utilised in the current study were internal (ILoC) and external (health professionals locus of control - EHPLoC) which were reliable for the current sample ( $\alpha=0.64$ for EHPLoC and 0.70 for ILoC).

6. Psychological Well-Being (W-BQ12) (Bradley, 1994). The W-BQ12 is widely used, particularly in clinical trials (Bradley \& Lewis, 1990; Witthaus, Stewart, \& Bradley, 2001). The W-BQ12 is designed to be used with populations suffering from chronic illnesses. The W-BQ12 (Bradley, 1994) discriminates between factors which are related to chronic illness and psychological status.

This subscales measure negative well-being, energy, positive and general wellbeing. This measure was reliable for the sample of this study (W-B12 $-\alpha=.80$ ).

The authors employed a cross-sectional design.

\subsection{Procedure}

Prospective participants received a pack containing a consent form, the questionnaires and a self-addressed prepaid envelope. Participants' personal details were transformed into codes before analysis to ensure confidentiality was preserved.
The completion of the questionnaires took approximately 45 minutes.

\subsection{Statistical Analysis}

Authors used Multiple Regression analyses to test the main research questions/hypotheses. Correlations between $\mathrm{HbA} 1 \mathrm{c}$ for exploratory analyses were also carried out. An a-priori power calculation was conducted. Significance was considered at $\alpha=0.05$. For the $\mathrm{F}$ test of the multiple $\mathrm{R}^{2} \mathrm{a}$ large effect size of $f^{2}=0.35$ was expected (Edelstein \& Linn, 1985; Anderson et al., 1990; Schwarz et al., 1991; Plowright, \& Hirsch; 2002; Woodcock, Bradley, 2002; Harris., \& Mertlich, 2003; Speight, 2003)

For an analysis with four independent variables for the main hypotheses, based on Cohen's d, a sample of 39 participants would be required to achieve a power of 0.80 .

Statistical Package for Social Science (SPSS) version 14.0 was used to analyse the data. All tests were 2-tailed unless otherwise stated.

\section{Results}

\subsection{Participants Characteristics}

Participant' socio-demographics characteristics, medical information and diabetes regimens are indicated in tables 1 .

\subsection{Correlations}

Correlations were conducted between $\mathrm{Hba} 1 \mathrm{C}$ and the psychological constructs. HbA1C levels were obtained for 34 participants. A few of participants failed to attend hospital or GP appointments for over 90 days and accordingly, $\mathrm{HbA1C}$ levels recorded had to be disregarded following DEP (2007) guidelines that it might reflect their current glycaemic control. A few HbA1c levels were obtained retrospectively from General Practitioners (GPs).

There was a significant negative correlation between $\mathrm{HbA1C}$ and external locus of control (health professionals) beliefs. People with higher HbA1C had lower external HP beliefs $(r(34)=-.47 ; p<.05)$. Participants with lower internal locus of control beliefs had higher HbA1C $(\mathrm{r}(34)=-.48$; $\mathrm{p}<.05)$. Lower psychological wellbeing was also negative correlated with $\mathrm{HbA1C}(\mathrm{r}(34)=-.52 ; \mathrm{p}<.05)$.

There was a positive correlation between negative wellbeing and $\mathrm{HbA1C}$, that is higher levels of negative well-being were associated with higher $\mathrm{HbA1C}$

$(\mathrm{r}(34)=.46 ; \mathrm{p}<.05)$.

\subsection{Multiple Regressions}

A standard multiple regression was performed with diabetes knowledge, internal and external locus of control beliefs as independent variables and general wellbeing as the dependent variable (table 2.1). These variables accounted for a significant amount of variance in psychological wellbeing $\left(\mathrm{R}^{2}=.404\right.$, adjusted $\mathrm{R}^{2}=.351 ; \mathrm{F}(3,42)=7.674$, $\mathrm{p}<0.000$ ). The partial regression coefficients showed that all 
variables made unique significant contributions to the model: Internal locus of control $(\mathrm{B}=.545, \beta=.332$, $\mathrm{t}(42)=2.393, \mathrm{p}<.05)$. and external locus of control $(\mathrm{B}=.614$, $\beta=.367, \mathrm{t}(42)=2.607, \mathrm{p}=.01$ ) each made a unique significant contribution to the model Diabetes knowledge was also independently associated with wellbeing after controlling for locus of control $(\mathrm{B}=.212, \beta=.347, \mathrm{t}(42)=2.559 \mathrm{p}<.05$, $\mathrm{p}>.05)$. Therefore, the model showed a highly significant relationship to the $\mathrm{DV}$, and this was carried by a combination of the variables and individually all three variables made significant independent contributions to the model.

\section{Discussion}

This study aimed to explore the nature of the relationship between psychological wellbeing, diabetes knowledge and locus of control amongst young adults with type 1 diabetes. A combination of internal and external locus of control beliefs and diabetes knowledge indicate psychological wellbeing.

\subsection{Locus of Control and Glycaemic Control}

The current study found that participants who held lower beliefs in their internal ability to control their diabetes had higher levels of HbA1C. People with an internal of locus of control would be more likely to adhere to their treatment regimen as they would expect their diabetes to be controlled by their actions (Bradley, 1994). Clearly, people who have hyperglycaemic episodes are less likely to feel in control of their diabetes. These findings have been corroborated by a number of previous studies: A study by Maltby and colleagues (2007) for example, found an association between internal locus of control, good diabetes management and improved physical and mental health. The current study also found that higher levels of HbAlc were associated with lower beliefs in health professionals exerting control over one's diabetes. This will be discussed next in this section.

\subsection{HbA1c and External Locus of Control}

External locus of control beliefs are normally associated with poorer compliance. For instance a study (Schlenk \& Hart, 1984). found that in adults with type 1 diabetes, external locus of control was associated both with poor short-term control at baseline and prediction of poorer long-term control over time. Conversely, other studies have supported the link between good metabolic control and external locus of control beliefs (Bradley, 1994, Matby et al., 2007; Arraras, 2002; Elfström \& Kreuter, 2006). Holding beliefs in external locus of control (health professionals) and internal locus of control can be beneficial: the combination of internal and powerful others locus of control is considered useful: while the patient feels personal responsibility for self-care, there is also a good patient-provider relationship. A recent meta-analysis (Hummer, Vannatta, \& Thompson, 2011) reviewing the association between beliefs related to diabetes and adherence to diabetes regimens corroborated these findings. They found that perceived positive relationship with one's physician as well as holding beliefs about personal consequences of adherence were associated with adherence to treatment

\subsection{Main Hypotheses}

The current study illustrated that diabetes knowledge and a combination of internal and external locus of control beliefs accounted for a significant amount of variance in psychological wellbeing. Each one of these variables was independently associated with psychological wellbeing. The relationship between internal locus of control and psychological wellbeing is well supported in the literature but there has been little evidence that external locus of control contributes to psychological wellbeing. Findings suggest that knowledge about one's condition is beneficial and is predictive of wellbeing in conjunction to being able to manage one's own diabetes and feeling supported by health professionals. Clearly, without knowledge of their condition diabetics cannot self-care appropriately. Diabetes knowledge has also been linked with prevention of diabetes complications, which are directly linked with depression and anxiety amongst young adults with diabetes (Fonagy et al., 1987). Accordingly, it is not surprising that better knowledge of diabetes is also associated with higher levels of psychological wellbeing.

The current study shows that the profile of the well adjusted young adult with type 1 diabetes is high in internal and/or external (health professionals) locus of control beliefs and is knowledgeable about his/her condition. The implications of this finding to clinical practice will be discussed later in this section.

The current study attempted to address some of the methodological limitations of previous studies by using diabetes-specific measures to explore psychological constructs that have shown to be associated with diabetes management in previous studies.

\section{Conclusions}

This study sheds light on the importance of addressing health professionals-patients communication: Given that external health professionals locus of control is a strong predictor of psychological wellbeing, it is important to address patients' expectations of their health practitioners to ascertain they are realistic. Also, since diabetes knowledge is also associated with wellbeing, it would be appropriate for health practitioners to provide education to their patients which is comprehensive, tailored to their specific needs, clear and up-to-date. Education interventions for diabetes patients are relatively straight forward and a number of studies demonstrated it has a significant positive effect on a number of domains including depression, self-care behaviours, self-efficacy. It also increases health professionals and patients self-awareness and improves health care provider-patient relationship. Patient education can increase 
diabetes knowledge empowering patients and their carers to take informed decisions about the type of regimen they want to follow. This can only take place if patients and carers can realistically evaluate some of their perceived barriers and factors that would facilitate self-care in relation to their elected regimen. A collaborative decision making process is likely to empower patients and increase their adherence to treatment, perceived self-efficacy, internal locus of control beliefs, quality of life and wellbeing. This type of intervention may also help patients to feel involved in their care, which might result in a decrease in the number of patients lost to follow-up.

\section{REFERENCES}

[1] International Diabetes Federation (2009). IDF Diabetes Atlas (4th ed.). Brussels: Belgium.

[2] The Department of Health (2007). Making every young person with diabetes matter. Report of children and young people with diabetes working group, 1-76.

[3] National Institute of Clinical Excellence. (2004). Type 1 diabetes in adults. National clinical guideline for diagnosis and management in primary and secondary care.

[4] The Department of Health (2003). National Service Framework for Diabetes: Delivery Strategy, 1-32

[5] Wolpert, H. A., \& Anderson, B. J. (2001). Metabolic control matters: Why is the message lost in the translation? The need for realistic goal-setting in diabetes care. Diabetes Care, 27(7), $1301-1303$.

[6] Bradley, C. (1996). Measuring Quality of Life. The Diabetes Annual, 10, 207-224.

[7] The National Diabetes Education Program (NDEP) (2007). The National Diabetes Education Program Control Your Diabetes. 13(4), 65-82.

[8] Woodcock A, Bradley C, Plowright R and Hirsch A (2002) How does diabetic retinopathy affect quality of life? Interviews to guide the design of a condition-specific, individualised questionnaire: the RetDQoL. Diabetic Medicine, 19 (2), 98-99.

[9] Bradley, C. (1994). Measures of perceived control of diabetes. In C. Bradley (Ed.) Handbook of Psychology and Diabetes: A guide to psychological measurement in diabetes research and practice. Chur, Switzerland: Harwood Academic Publishers.

[10] González, M., Casas, F. \& Coenders, G. (2007): A Complexity Approach to Psychological Well-Being in Adolescence: Major Strengths and Methodological Issues. Social Indicators Research, 80, 267-295.

[11] Jacobson A. (1993). Depression in adults with diabetes: an epidemiological evaluation. Diabetes Care, 16, 1621-1623.

[12] Kakleas K., Kandyla B., Karayianni C., Karavanaki K. (2009). Psychosocial problems in adolescents with type 1 diabetes mellitus. Diabetes and Metabolism, 35(5), 339-350.

[13] Kluding P.M., Singh R., Goetz J., Rucker J., Bracciano S.,
Curry N. (2010). Feasibility and effectiveness of a pilot health promotion program for adults with type 2 diabetes: Lessons learned. Diabetes Educator. 36(4), 595-602.

[14] Castillo A., Giachello A., Bates R., Concha J., Ramirez V., Sanchez C., Pinsker E., Arrom J. (2010). Community-based diabetes education for latinos: The diabetes empowerment education program. Diabetes Educator. 36(4), 586-594).

[15] UK Prospective Diabetes Study Group. (1998). Tight blood pressure control and risk of macrovascular and microvascular complications in Type 2 diabetes. The Lancet, 317(38), 703-713

[16] Coleman K.J., Ocana L.L., Walker C., Araujo R.A., Gutierrez V., Shordon M., Oratowski-Coleman J., Philis-Tsimikas A (2010). Outcomes from a culturally tailored diabetes prevention program in Hispanic families from a low-income school: Horton Hawks Stay Healthy (HHSH). Diabetes Educator, 36(5), 784-792.

[17] Anderson, B. J., \& Rubin, R. R. (1996). Practical Psychology for Diabetes Clinicians (1st ed.). Alexandria: The American Diabetes Association.

[18] Walker. N. (2004). Family Functioning and Diabetic Ketoacidosis in Pediatric Patients with Type 1 Diabetes. M.Sc. thesis. University of Florida

[19] Ogden, J. (2001). Health Psychology: A textbook (2nd ed.). Buckingham, UK; Philadelphia: Open University Press.

[20] Maltby, J., Day, L., \& Macaskill, A. (2007). Personality, Individual Differences and Intelligence. Harlow: Pearson Prentice Hall.

[21] Gherman A., Schnur J., Montgomery G., Sassu R., Veresiu I. David D. (2011). How are adherent people more likely to think? A meta-analysis of health beliefs and diabetes-care. Diabetes Educator. 37(3), 392-408

[22] Arraras, J. I., Wright, S. J., Jusue, G., Tejedor, M., \& Calvo, J. I. (2002). Coping style, locus of control, psychological distress and pain-related behaviours in cancer and other diseases. Psychology, Health \& Medicine, http://www. informaworld.com/smpp/35862140-37011873/title $\sim$ content $=\mathrm{t} 713441$ $652 \sim \mathrm{db}=\mathrm{all} \sim \mathrm{tab}=\mathrm{issueslist} \sim \mathrm{branches}=7-\mathrm{v} 77(2), 181-187$.

[23] Elfström, M., \& Kreuter, M. (2006). Relationships between locus of control, coping strategies and emotional well-being in persons with spinal cord lesion. Journal of Clinical Psychology in Medical Settings, 13(1), 89-100.

[24] Speight, J., \& Bradley, C. (2001). The ADKnowl: Identifying knowledge deficits in diabetes care. Diabetic Medicine, 18, 626-639.

[25] Bradley, C. (1994). The Well-being+ Questionnaire. In C. Bradley (Ed.), Handbook of Psychology and Diabetes: A guide to psychological measurement in diabetes research and practice (pp. 89-109). Chur, Switzerland: Harwood Academic.

[26] Bradley, C., \& Lewis, K. S. (1990). Measures of psychological well-being and treatment satisfaction developed from the response of people with tablet-treated diabetes. Diabetic Medicine, 7, 445-451.

[27] Witthaus, E., Stewart, J., \& Bradley, C. (2001). Treatment satisfaction and psychological well-being with insulin glargine compared with NPH in patients with Type 1 diabetes. 
Diabetic Medicine, 18, 619-625.

[28] Speight, J. (2003). Development of knowledge and quality of life measures for improving diabetes care. Ph.D. thesis. University of London.

[29] Woodcock A, Bradley C, Plowright R and Hirsch A (2002) How does diabetic retinopathy affect quality of life? Interviews to guide the design of a condition-specific, individualised questionnaire: the RetDQoL. Diabetic Medicine, 19 (2), 98-99

[30] Anderson, B. J., Auslander, W. F., Jung, K. C., Miller, J. P., \& Santiago, J. V. (1990). Assessing family sharing of diabetes responsibilities. Journal of Pediatric Psychology, 15, 477-492.

[31] Harris, M. A., \& Mertlich, D. (2003). Piloting home-based family systems therapy for adolescents with poorly controlled diabetes. Child. Health Care, 32(1), 65-79.35. Pouwer F, Snoek FJ, van der Ploeg HM, Adèr HJ and Heine RJ (2000) The Well-being Questionnaire: evidence for a three-factor structure with 12 items (W-BQ12). Psychological Medicine, $30,455-462$.

[32] Schwarz, L. S., Coulson, L. R., . Toovy, D., Lyons, L. S., Flaherty, J. A. (1991). Perception and utilization of social support in diabetic control. Diabetes Research and Clinical Practice, 41(3), $207-211$.

[33] Edelstein, J. and Linn, M. W. (1985). Locus of control and the control of diabetes. The Diabetes Educator, 13(1), 51-54.

[34] Schlenk E. And Hart, L (1984). The Relationship between Health Locus of Control, Health Value, and Social Support \& Compliance of Persons with Diabetes Mellitus. Diabetes Care, 7(6), 566-574.

[35] Hummer K. Vannatta J. Thompson D. (2011). Diabetes Educator. 37(1),104-10

[36] Fonagy, P., Moran, G. S., Lindsay, M. K. M., Kurtz, A. B., \& Brown, R. (1987). Psychological adjustment and diabetic control. Arch. Dis. Child., 62, 1009-1113 